in $29.2 \%$ and $18.8 \%$, respectively. Best objective response rate was $20.8 \%$ and disease control rate was $66.6 \%$. Median progression-free survival (PFS) was 5.3 (95\% confidence interval=3.9-6.6), respectively. The median number of cycles for PEMBRO+LEN was 4.5. More than half of patients (56.2\%) received LEN dose reduction was once or more but it did not affect survival outcomes. Common AEs of LEN was fatigue $(18.8 \%)$, hypertension $(16.7 \%)$ and hypothyroidism (14.6\%). Treatment-free interval (TFI) was the only statistically significant factor associated with PFS $(\mathrm{p}=0.017)$. In a subgroup analysis, TFI 5 months showed statistically higher in best overall response $(\mathrm{p}=0.042)$ and in the number of the cycle of AEs first encountered during PEMBRO+LEN treatment ( $\mathrm{p}=0.017)$.

Conclusion: In Korean patients with recurrent endometrial cancer, PEMBRO+LEN treatment showed acceptable response rates with tolerable AEs when treatment was supported with optimal LEN dose reduction.

Oral (OM2)

Endometrial Hyperplasia, Endometrial Intra-epithelial Neoplasia, and Endometrial Cancer

https://doi.org/10.3802/jgo.2021.32.S1.0M2

\section{Menopausal status combined with serum CA125 level significantly predicted concurrent endometrial cancer in women diagnosed with atypical endometrial hyperplasia before surgery}

\section{Yaochen Lou, Jiongbo Liao, Jun Guan*}

Obstetrics and Gynecology Hospital of Fudan University, Shanghai, China (junguan2018@163.com)

Objective: About $10 \%-60 \%$ of patients with atypical endometrial hyperplasia diagnosed before surgery (preoperative-AEH) are found to have concurrent endometrial cancer (EC) at definitive hysterectomy, leading to incomplete primary surgery and delayed adjuvant treatment. This study aims to investigate the potential risk factors of concurrent EC in preoperative-AEH patients in a clinical setting with gynecological pathology review.

Methods: All patients diagnosed with $\mathrm{AEH}$ by endometrial biopsy or curettage and then underwent definitive hysterectomy from January 2016 to December 2019 were analyzed. All diagnoses were reviewed by gynecological pathologists. Results: Totally 624 preoperative-AEH patients were included, $30.4 \%$ of whom had concurrent EC. In multivariate analysis, postmenopausal status and cancer antigen 125 (CA125) $\geq 35$ $\mathrm{U} / \mathrm{mL}$ significantly correlated with concurrent EC (odds ratio
$[\mathrm{OR}]=3.33,95 \%$ confidence interval $[\mathrm{CI}]=1.76-6.30 ; \mathrm{OR}=2.12$, $95 \% \mathrm{CI}=1.13-3.98)$. Similar findings were showed in predicting intermediate-high-risk EC. The combined predicting value of postmenopausal status and CA125 $\geq 35 \mathrm{U} / \mathrm{mL}$ were remarkably increased for concurrent $\mathrm{EC}(\mathrm{OR}=14.10,95 \% \mathrm{CI}=1.59-125.22)$ and intermediate-high-risk EC $(\mathrm{OR}=30.2$, 95\% $\mathrm{CI}=2.44-$ 374.40). Notably, concurrent intermediate-high-risk EC was more commonly seen in preoperative-AEH women with postmenopausal time $\geq 5$ years $(\mathrm{OR}=7.83,95 \% \mathrm{CI}=2.44-25.18$, $\mathrm{p}=0.001$ ), with no difference in this risk among subgroups of premenopausal status, postmenopausal time $<2$ years or between 2-5 years.

Conclusion: Preoperative-AEH patients with postmenopausal status and elevated level of CA125 may have high risk of concurrent EC. Adequate pre-surgical evaluation and sentinel lymph node sampling should be suggested for such patients.

Oral (OM3)

Endometrial Hyperplasia, Endometrial Intra-epithelial Neoplasia, and Endometrial Cancer

https://doi.org/10.3802/jgo.2021.32.S1.0M3

\section{Clinical implications of neoadjuvant chemotherapy in advanced endometrial cancer: a multi-center retrospective cohort study}

\author{
Hyunji Lim, ${ }^{1}$ Se Ik Kim, ${ }^{1}$ Tae Hun Kim, ${ }^{2}$ Dong Hoon Suh, ${ }^{3}$ \\ Myong Cheol Lim, ${ }^{4}$ Jae-Weon Kim ${ }^{1, *}$ \\ 'Seoul National University College of Medicine, Seoul, Korea \\ (kjwksh@snu.ac.kr) \\ ${ }^{2}$ Seoul Metropolitan Government-Seoul National University Boramae \\ Medical Center, Seoul, Korea \\ ${ }^{3}$ Seoul National University Bundang Hospital, Seongnam, Korea \\ ${ }^{4}$ National Cancer Center, Goyang, Korea
}

Objective: In a portion of patients with advanced endometrial cancer, upfront complete cytoreduction is not possible. Hence, we aimed to assess the feasibility and effectiveness of neoadjuvant chemotherapy followed by interval debulking surgery (NAC-IDS) in unresectable, metastatic endometrial cancer. We also investigated factors which related to the favorable conditions for NAC-IDS in endometrial cancer. Methods: From the four institutions in Korea, we identified stage IIIC-IVB endometrial cancer patients who received NACIDS between 2008 and 2020. We collected patients' baseline characteristics, NAC regimens, response to NAC, and surgical details. Both progression-free survival (PFS) and overall survival (OS) were calculated, and factors affecting survival outcomes were investigated. 
Results: In total, 32 patients were included: endometrioid $(n=18)$, serous $(n=5)$, carcinosarcoma $(n=6)$, and others $(n=3)$. Among them, 25 (78.1\%) had stage IVB disease. The most common NAC regimen was paclitaxel-carboplatin $(n=25$, $78.1 \%$ ), administered with median of 6 cycles. While 26 $(81.3 \%)$ showed an objective response, 2 (6.3\%) progressed despite NAC. At the time of IDS, 23 (71.9\%) achieved complete cytoreduction. During 30.5 months of the median followup, there were 23 recurrences and 7 deaths, corresponding to 19 months of median PFS and $46.7 \%$ of 3 -year OS rate. In multivariate analysis, endometrioid histology was associated with better PFS (hazard ratio $[H R]=0.181 ; \mathrm{p}=0.001$ ) and OS (HR=0.112; $\mathrm{p}=0.029$ ), while complete cytoreduction significantly improved PFS (HR=0.258; $\mathrm{p}=0.008)$. Conclusion: We found NAC-IDS is feasible and effective in unresectable, metastatic endometrial cancer. Tumor histology and the possibility of complete cytoreduction would be the primary considerations for NAC-IDS.

Oral (OM4)

Endometrial Hyperplasia, Endometrial Intra-epithelial Neoplasia, and Endometrial Cancer

https://doi.org/10.3802/jgo.2021.32.S1.0M4

\section{Comparison of sentinel lymph node biopsy alone to complete lymphadenectomy in endometrial cancer patients: a retrospective study}

\section{Amany Abdelwadoud Makroum, ${ }^{1, *}$ Jung-Yun Lee, ${ }^{2}$ Eun Ji Nam, ${ }^{2}$ Sunghoon Kim, ${ }^{2}$ Sang Wun Kim, ${ }^{2}$ Young Tae Kim ${ }^{2}$ \\ 'Department of Obstetrics and Gynecology, Faculty of Medicine, Mansoura University, Mansoura, Egypt (amany_makroum@mans.edu.eg) ${ }^{2}$ Department of Obstetrics and Gynecology, Institute of Women's Life Medical Science, Yonsei University College of Medicine, Seoul, Korea}

Objective: Sentinel lymph node (SLN) biopsy allows to spare node negative patients from surgical comorbidities. Our objectives were evaluation of outcomes of SLN biopsy only compared to additional lymph node (LN) dissection in endometrial cancer patients.

Methods: Single institutional retrospective study of endometroid type of endometrial carcinoma patients at Yonsei Cancer Center who underwent minimally invasive surgical staging with SLN biopsy with or without additional LN dissection between January 2015 to December 2019.

Results: A total of 301 patients were included in this study. Eighty-two underwent SLN biopsy only and were compared to 219 with extra LN dissection. The median age was 53 years and median body mass index was $25 \mathrm{~kg} / \mathrm{m}^{2}$. In terms of operative outcomes, SLN biopsy only group showed statistically significant decreased surgical time $(\mathrm{p}=0.001)$. However, estimated blood loss during operation did not show any statistically significant difference. The sites and numbers of SLN did not show statistically significant difference. There was one case with LN metastasis from whom SLN metastasis was not detected. This case reflects false negativity of SLN biopsy and overall false negative rate of SLN biopsy was $0.45 \%$. Recurrence and death events did not show any statistically significant difference between the two groups during the follow-up period. Conclusion: This study showed similar prognosis among the 2 included groups with acceptable diagnostic accuracy of SLN biopsy with small detected false negative rates. Thus, LN assessment can be considered as valuable and could safely replace $\mathrm{LN}$ dissection.

Oral (OM5)

Endometrial Hyperplasia, Endometrial Intra-epithelial Neoplasia, and Endometrial Cancer

https://doi.org/10.3802/jgo.2021.32.S1.0M5

\section{The fluorescence imaging for laparoscopic and laparotomic endometrial sentinel lymph node biopsy (FILLES) trial: Siriraj Gynecologic sentinel Node of endometrial cancer (SiGN-En) study}

\section{Khemanat Khemworapong, "Atthapon Jaishuen, Mongkol Benjapibal, Perapong Inthasorn, Boonlert Viriyapak, Pisutt Srichaikul, Nida Jareemit, Malee Warnnissorn, Suchanan Hanamornroongruang \\ Mahidol University, Nakhon Pathom, Thailand (khemanatkhem@gmail.com)}

Objective: To establish the detection rate of sentinel lymph node (SLN) biopsies and to determine the sensitivity and false-negative rate of SLN biopsies compared with those of systematic pelvic and para-aortic lymphadenectomies in endometrial cancer.

Methods: This prospective cohort study enrolled patients with endometrial cancer who were scheduled for surgical staging. Patients with a history of chemotherapy or radiotherapy, an abnormal liver function test, or an allergy to indocyanine green (ICG) were excluded. All patients underwent surgical staging with an ICG injection at the cervix. SLNs were identified by a near-infrared fluorescent camera. All SLNs were sent to a pathologist for ultra-staging.

Results: From November 2019 to March 2021, 101 patients underwent SLN mapping and surgical staging. SLNs were not detected bilaterally in 2 patients. The detection rate of the SLN biopsies in this study was $97.9 \%$. Thus, the accuracy of the SLN biopsies was $98.9 \%$. The sensitivity for finding metastatic SLNs 